\title{
Detection of Angiostrongylus vasorum by quantitative PCR in bronchoalveolar lavage fluid in Belgian dogs
}

\author{
A.-M. Canonne*, E. Roels*, Y. Caron†, B. Losson†, G. Bolen*, I. Peters $\ddagger$, F. Billen* and C. Clercx* \\ *Department of Clinical Sciences, Faculty of Veterinary Medicine, University of Liège, 4000 Liège, Belgium \\ $\dagger$ Department of Infectious and Parasitic Diseases, Faculty of Veterinary Medicine, University of Liège, 4000 Liège, Belgium \\ ¥Torrance Diamond Diagnostic Services, The Innovation Centre, University of Exeter, Exeter, Devon EX4 4RN
}

Овлестіves: To describe Angiostrongylus vasorum infection in a series of clinical cases over a 12-month period, report the use of quantitative PCR on broncho-alveolar lavage fluid and investigate the possibility of previously undiagnosed angiostrongylosis in a retrospective cohort of coughing and healthy dogs.

Methods: Pulmonary angiostrongylosis was diagnosed based on compatible clinical signs and positive quantitative PCR on broncho-alveolar lavage fluid and/or positive Baermann examination. Quantitative PCR was also performed retrospectively on broncho-alveolar lavage fluid from 65 dogs (55 coughing and 10 healthy dogs), examined between 2008 and 2014; results of Baermann examination were also available in 33 dogs.

Results: Seven young adult dogs from Southeastern Belgium with respiratory clinical signs were diagnosed with angiostrongylosis between March 2013 and April 2014. Positive broncho-alveolar lavage fluid quantitative PCR results and positive Baermann examination were obtained in 5/5 and 2/5 dogs, respectively. In the remaining two dogs, only Baermann analysis was performed. Among the retrospective cohorts, only one broncho-alveolar lavage fluid from a coughing dog was quantitative PCR-positive whereas all faecal samples were negative.

Clinical Significance: Until recently, canine angiostrongylosis was not reported in Belgium. It should now be included in the differential diagnosis of coughing Belgian dogs. Identification of affected dogs may be aided by quantitative PCR on broncho-alveolar lavage fluid.

Journal of Small Animal Practice (2016) 57, 130-134

DOI: $10.1111 /$ jsap.12419

Accepted: 3 October 2015; Published online: 25 November 2015

\section{INTRODUCTION}

Angiostrongylus vasorum (A. vasorum) is a metastrongyloid nematode inhabiting the right cardiac ventricle and the pulmonary arteries of dogs and other wild canids such as the red fox (Vulpes vulpes). Parasite infestation can cause coagulopathy and respiratory signs as well as cardiovascular, central nervous system and, rarely, digestive tract disorders, either singly or in combination (Bolt et al. 1994, Chapman et al. 2004, Jeffery et al. 2004, Bourque et al. 2008, Koch \& Willesen 2009, Helm et al. 2010 ,
Gredal et al. 2011, Elsheikha et al. 2014). The condition may be fatal if left untreated, particularly in young dogs with a high worm burden and coagulation disorders (Yamakawa et al. 2009, Gredal et al. 2011).

Recent studies highlight an increasing prevalence of this parasitic infection in long-standing endemic areas and an expanding distribution of the parasite in both Europe and North America (Bourque et al. 2008, Yamakawa et al. 2009, Helm et al. 2010, Conboy 2011, Gredal et al. 2011, Gallagher et al. 2012, Traversa et al. 2013). In Germany, a neighbouring country of Belgium, 
epidemiological surveys have shown a constantly increasing prevalence of faecal detection of the parasite in dogs between 2003 and 2009, particularly in southwestern areas (Barutzki \& Schaper 2009, Taubert et al. 2009). Recently, autochthonous infections of $A$. vasorum were reported in dogs in The Netherlands (Van Doorn et al. 2009).

Faecal detection of the first-stage larvae (L1) is the most frequently used method to diagnose $A$. vasorum infection (Bolt et al. 1994, Chapman et al. 2004, Koch \& Willesen 2009, Elsheikha et al. 2014). A direct faecal smear can be easily used in general practice, however, despite its attractive potential for rapid diagnosis of severely affected dogs, its sensitivity is poor (Humm \& Adamantos 2010). Baermann examination on three consecutive faecal samples is thus considered to be more sensitive. Nevertheless, despite its high specificity, the sensitivity of this test is questionable, mainly because of irregular faecal shedding of L1 larvae (Bolt et al. 1994, Oliveira-Junior et al. 2006, Elsheikha et al. 2014); indeed, larvae have been detected by broncho-alveolar lavage in experimentally infected dogs before faecal detection by Baermann examination (Barçante et al. 2008). Therefore, diagnostic techniques more sensitive than faecal examination are needed. Detection of specific antibodies by ELISA assays is highly sensitive but not commercially available and only used for epidemiological studies (Schucan et al. 2012, Guardone et al. 2013, Schnyder et al. 2013). Parasite circulating antigens can be detected in whole blood using a highly specific and sensitive sandwich-ELISA assay which has been documented to be a valid alternative for early, reliable diagnosis and for follow-up after treatment (Verzberger-Epshtein et al. 2008, Schnyder et al. 2011). A new in-house rapid device for detection of highly specific circulating antigen is now commercially available in several European countries and been recently evaluated. High sensitivity $(84 \%)$ was found in a population of 39 naturally infected dogs when Baermann examination of faeces was considered as the gold standard (Angio Detect ${ }^{\mathrm{TM}}$ Test, Idexx Laboratories, Westbrook, ME, USA, Schnyder et al. 2014). However this study provides no information about infected cases that are Baermann-negative and also reported lower sensitivity when compared to the Baermann test for experimentally infected cases.

Lastly, quantitative PCR (qPCR) tests have recently been made commercially available and appear promising, although, so far, qPCR has been only anecdotally performed on broncho-alveolar lavage fluid samples (Jefferies et al. 2011). Until now, the presence and the prevalence of $A$. vasorum infection among coughing dogs have not been investigated in Belgium although a fatal neurological case was reported very recently (Jolly et al. 2014). The aims of the present study were: (1) to describe the clinical use of qPCR on broncho-alveolar lavage fluid in a series of dogs diagnosed with pulmonary angiostronglosis over a 12-month period and (2) to investigate a posteriori the possibility of previously undiagnosed angiostrongylosis among a series of coughing and healthy dogs using both newly available qPCR on collected and stored broncho-alveolar lavage specimens and Baermann examination of faeces.

\section{MATERIALS AND METHODS}

\section{Clinical series}

Client-owned dogs presented with respiratory clinical signs at the Liège University Veterinary Small Animal Teaching Hospital between March 2013 and April 2014 and diagnosed with pulmonary angiostrongylosis were prospectively recruited.

Pulmonary angiostrongylosis was suspected based on compatible respiratory clinical signs, radiologic findings, bronchoscopy and cytological examination of broncho-alveolar lavage fluid; diagnosis was definitively confirmed by a positive result of qPCR on broncho-alveolar lavage fluid and/or a faecal detection of L1 larvae by Baermann examination. In two dogs, only faecal samples were available because bronchoscopy was not performed owing to financial limitations.

Bronchoscopy, bronchoalveolar lavage and laboratory processing of broncho-alveolar lavage fluid were performed as previously described in detail (Dehard et al. 2008). All dogs were anaesthetised, using various anaesthetic protocols. Premedication with intramuscular or intravenous administration of medetomidine $\left(5\right.$ to $10 \mu \mathrm{g} / \mathrm{kg}$, Sedator ${ }^{\odot}$, Eurovet Animal Health, Bladel, Netherlands) and/or butorphanol ( 0.2 to $0.3 \mathrm{mg} / \mathrm{kg}$, Dolorex ${ }^{\odot}$, Intervet Int. via MSD Animal Health, Boxmeer, Netherlands), and/or midazolam $\left(0.3 \mathrm{mg} / \mathrm{kg}\right.$, Dormicum ${ }^{\odot}$, Roche Laboratoire, Anderlecht, Belgium) was followed by IV administration of propofol up to $6 \mathrm{mg} / \mathrm{kg}$ for induction, and with boluses of 0.1 to $0.2 \mathrm{mg} / \mathrm{kg}$ for maintenance (Diprivan ${ }^{\odot}$, AstraZeneca, Uccle, Belgium). In most dogs, a 5-minute preoxygenation period was used. Animals were initially not intubated: oxygen saturation was controlled during the procedure and subsequent recovery using continuous pulse oximetry monitoring. All dogs received supplementary oxygen by flow-by; two of them were intubated during or after the bronchoscopy due to moderate hypoxemia.

Broncho-alveolar lavage fluid analysis consisted of total cell count determination (Thomas' cell), manual differential cell counts calculation, cytological examination of cyto-centrifugated smears and semi-quantitative bacterial culture. qPCR analysis was performed on uncentrifugated specimens. Genomic DNA (gDNA) was purified from $200 \mu \mathrm{L}$ of uncentrifugated bronchoalveolar lavage fluid using the DNeasy Blood and Tissue Kit (QIAGEN, Manchester, UK), with the DNA eluted in $100 \mu \mathrm{L}$, and qPCR analysis performed on $5 \mu \mathrm{L}$ gDNA as described previously (Jefferies et al. 2011).

Baermann examination was performed on three consecutive defaecations in five of seven dogs, as previously described (Forey 1989). Faecal samples were stored at $4^{\circ} \mathrm{C}$ after collection, and then processed immediately by the laboratory upon receipt.

\section{Retrospective study in coughing and healthy dogs}

In order to retrospectively investigate the presence of the parasite among coughing and healthy dogs and evaluate whether or not we previously failed to diagnose pulmonary angiostrongylosis, newly available qPCR analysis was a posteriori performed on 65 broncho-alveolar lavage fluid samples collected between January 2008 to April 2014 from 55 coughing dogs and 10 healthy dogs. 
At the time of broncho-alveolar lavage fluid collection in both healthy and coughing dogs, Baermann examination was also performed in 33 of them; faecal samples were collected over one (healthy dogs and some coughing dogs) or three consecutive defecations (majority of coughing dogs) and stored and processed as for clinical cases.

In the retrospective analysis of broncho-alveolar lavage fluid of coughing and healthy dogs, all collected samples were immediately processed in a standardised manner after withdrawal of a minimal amount of fluid for total cell calculation, cytospin preparation and bacterial culture, part of the collected fluid was centrifugated to obtain pelleted cells and supernatant. Supernatant and pellets were frozen directly at $-80^{\circ} \mathrm{C}$. For the retrospective qPCR analysis, the frozen pelleted cells were thawed and sent to the laboratory for analysis after re-suspension in a small volume of sterile saline $(0.9 \% \mathrm{NaCl})$ and analysed as the fresh samples.

\section{RESULTS}

\section{Clinical series}

Pulmonary angiostrongylosis was diagnosed in seven small- or medium-sized dogs with a median age of 2.3 years (range 0.4 to 9.0). All of them were living in Southern or Eastern Belgium and diagnosed over a 12-month period, between March 2013 and April 2014 and represented 3.1\% of the 225 registered canine respiratory consultations over this period of time. All were presented for daily cough and/or a moderate to severe exercise intolerance and dyspnoea. One dog also suffered from an acute central vestibular syndrome (direction-changing nystagmus, unilateral proprioceptive deficit). No dog had a history of travel to well-recognised endemic areas during the six preceding months.

Mild eosinophilia, neutrophilia and regenerative anaemia were occasionally observed (in 2, 2 and 1 dogs, respectively). All dogs had some radiological changes compatible with pulmonary angiostrongylosis (moderate to severe bronchointerstitial or alveolointerstitial pattern, often more pronounced in peripheral and/ or caudo-dorsal areas).

Bronchoscopy was performed in five dogs: all had diffuse mucosal congestion and oedema and blood-containing secretions originating from the main bronchi were observed in three dogs (Fig 1). No coagulation parameters were measured. Cytological examination of cytocentrifugated May-GrünwaldGiemsa-stained smears of broncho-alveolar lavage fluid showed neutrophilic (five dogs) or mixed neutrophilic and eosinophilic airway inflammation (two dogs) (Fig 2) and identified L1 larvae (Fig 3) in two dogs. There were no complications as a result of the bronchoalveolar lavage.

In five dogs, both broncho-alveolar lavage fluid and faeces were obtained: qPCR on broncho-alveolar lavage fluid was positive in all, at high or moderate level [cycle threshold $(\mathrm{Ct})$ between 26.7 and 29.6] while L1 larvae were detected in the faeces of only two animals despite collection of three samples over 3 days. In the remaining two dogs, no broncho-alveolar lavage fluid was collected, but Baermann analysis of faeces consecutively collected over 3 days was positive.

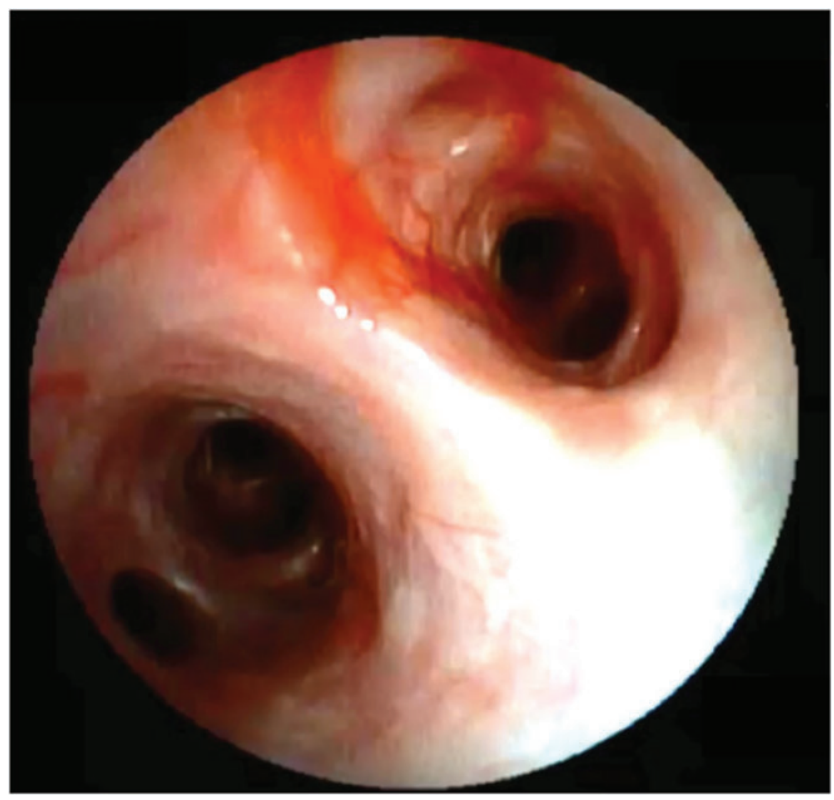

FIG 1. Bronchoscopic view of tracheobronchic bifurcation showing congestion of the tracheobronchial mucosa and blood-containing secretions from the left main bronchus

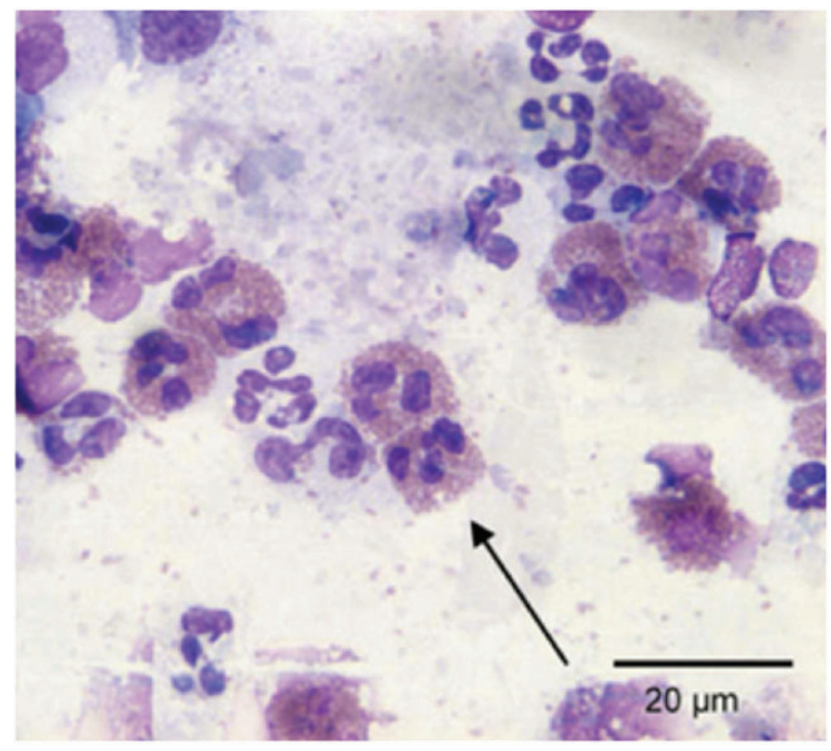

FIG 2. Eosinophilic (arrowed) airway inflammation detected in this cytospin sample of broncho-alveolar lavage fluid in one dog (MayGrünwald-Giemsa stain. $\times 1000$ magnification)

All dogs were treated with a 3-week course of fenbendazole $\left(50 \mathrm{mg} / \mathrm{kg} / \mathrm{day}\right.$, Panacur $^{\odot}$ Intervet International via MSD Animal Health) as classically advised (Koch \& Willesen 2009); in two dogs, two additional spot-on applications of moxidectin $\left(2.5 \mathrm{mg} / \mathrm{kg}\right.$, Advocate Spot-On ${ }^{\odot}$ Bayer, Diegem, Belgium) with a 1-month interval were prescribed because of persistent mild radiographical lesions after treatment with fenbendazole. In two dogs, anti-inflammatory doses of prednisolone $(0.5 \mathrm{mg} / \mathrm{kg}$ q24h, Prednisolone $^{\odot}$ Kela Laboratoria, Hoogstraten, Belgium) were prescribed for a short period of time (less than a week) because of the initial clinical severity in one $\operatorname{dog}$ and the worsening of 


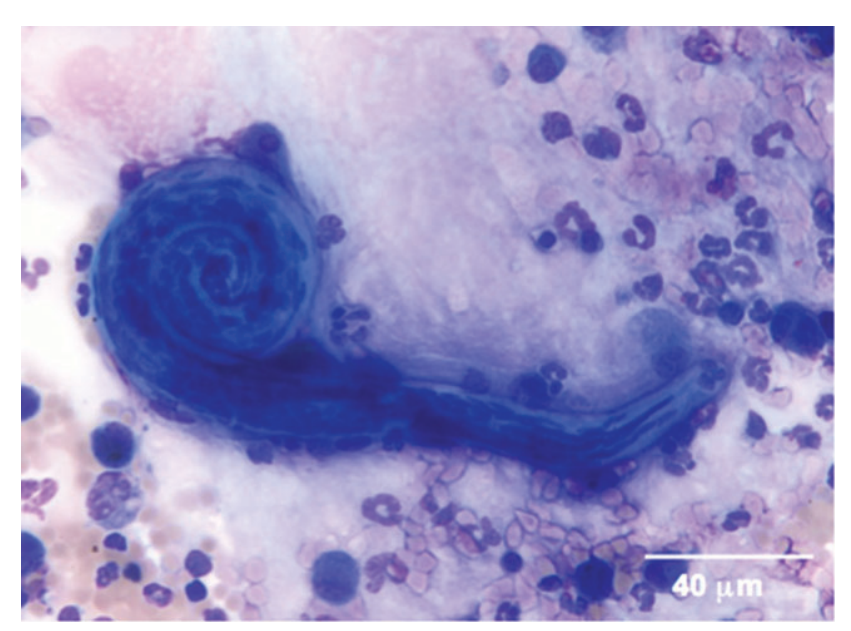

FIG 3. Cytological detection of Angiostrongylus vasorum L1 larva in broncho-alveolar lavage fluid from one dog (May-Grünwald-Giemsa stain. $\times 400$ magnification)

cough after initiation of the anthelminthic treatment in another. One dog was co-infected with Bordetella bronchiseptica diagnosed by both cytological observation of coccobacilli adhering to the cilia of epithelial cells of broncho-alveolar lavage fluid and positive qPCR on broncho-alveolar lavage fluid. Another dog was diagnosed with concomitant Crenosoma vulpis infestation, diagnosed by Baermann examination. Both of these dogs with bacterial and parasitic coinfection were treated accordingly (at the same time of the treatment for angiostrongylosis), with nebulised gentamicine (GENTA-kel 5\% ${ }^{\odot}$ Kela Laboratoria) and 4 weekly oral doses of milbemycin oxime $\left(1 \mathrm{mg} / \mathrm{kg}\right.$, Milbemax ${ }^{\odot}$ Novartis, Vilvoorde, Belgium). Clinical resolution was noticed in all dogs. No Baermann examination was available during the follow-up.

\section{Retrospective study in coughing and healthy dogs}

The healthy dogs were urban or suburban dogs with neither history nor clinical signs of respiratory problems. Broncho-alveolar lavage fluid had been previously collected and stored for earlier research studies for which ethical approval had been previously obtained from the Liege University local Ethical Committee; total cell counts and cytological examination were available and were within normal limits. Coughing dogs were diagnosed with various respiratory diseases such as eosinophilic bronchopneumopathy, B. bronchiseptica infection, bacterial bronchopneumonia and chronic bronchitis. qPCR was negative in the broncho-alveolar lavage fluid from all healthy dogs and yielded a positive result $(\mathrm{Ct}=22.0)$ in only one broncho-alveolar lavage fluid from a dog originally diagnosed with idiopathic eosinophilic bronchopneumopathy in 2011. In this dog, a short course of fenbendazole had been prescribed at the time of the initial diagnosis, despite a negative result of the Baermann analysis. Baermann examination was negative in all healthy and coughing dogs.

\section{DISCUSSION}

The present series represents the first clinical description of canine pulmonary angiostrongylosis in dogs presented with respiratory signs in Belgium. In this series of cases, diagnosis was essentially confirmed by qPCR on broncho-alveolar lavage fluid samples. Based on the recent cases diagnosed in Belgium (2013 to 2014) and the present retrospective investigation among coughing dogs over the last 7 years (2008 to 2014), it can be assumed that pulmonary angiostrongylosis is now an emerging disease in Belgium. Since its discovery in France in the middle of the 20th century (Doby et al. 1970), A. vasorum has been found to be endemic in several European and North American countries (Bolt et al. 1992, Bolt et al. 1994, Chapman et al. 2004, Conboy 2011). However, recent reports emphasise that the disease is spreading into several new geographical areas (Bourque et al. 2008, Yamakawa et al. 2009, Helm et al. 2010, Conboy 2011, Gredal et al. 2011, Gallagher et al. 2012, Traversa et al. 2013). Movements in animal populations, climatic global warming and changes in vector epidemiology are presumed to be mainly responsible for this spread (Morgan et al. 2009, Traversa et al. 2010). Seasonal breeding patterns and activity of the gastropod mollusc intermediate hosts (snails and slugs) are strongly sensitive to temperature and moisture (Morgan et al. 2009). In this respect, Belgium is part of a vast area in Western Europe considered highly suitable for the maintenance of $A$. vasorum life cycle. Furthermore, increasing densities of red foxes (Vulpes vulpes), the main wild reservoir of this nematode, in urban and periurban areas are likely to increase the risk for A. vasorum transmission to dogs (Bolt et al. 1992, Franssen et al. 2014). Based on results of previous studies, this nematode is increasingly detected in faeces of foxes from almost all countries neighbouring Belgium (Demiaszkiewicz et al. 2014, Elsheikha et al. 2014). Lastly, accessibility to accurate recently developed diagnostic methods [qPCR, immunochromatography test for serological in-clinic diagnosis, Angio Detect ${ }^{\mathrm{TM}}$ Test, Idexx Laboratories (Schnyder et al. 2014)] and heightened clinical awareness could also account for disease emergence.

Results of the present study support the use of qPCR as an adequate and reliable diagnostic technique to detect $A$. vasorum in broncho-alveolar lavage fluid in coughing dogs. In previous studies using qPCR analysis focused on analysis of blood or faeces, whereas broncho-alveolar lavage fluid was tested in only two dogs initially diagnosed through Baermann examination (Jefferies et al. 2011). The present series documents for the first time positive qPCR results on broncho-alveolar lavage fluid in Baermannnegative dogs. Additionally, in experimentally infected dogs, L1 larvae were found by cytological examination of broncho-alveolar lavage fluid in all dogs at 60 days post infection whereas only $85 \%$ of dogs had positive Baermann examination at the same time (Barçante et al. 2008). Therefore, broncho-alveolar lavage fluid might possibly allow detection of L1 larvae either by qPCR or by standard cytological examination before faecal larval shedding. Furthermore, broncho-alveolar lavage fluid can yield other useful diagnostic information, such as bacterial or parasitic coinfection, which may require specific or alternative therapeutic management. This was the case in two dogs in the present study. In addition, because none of the healthy dogs had positive qPCR results from broncho-alveolar lavage fluid the risk of false positive test results (from endoscope contamination for example) were considered unlikely. 
Inclusion criteria used in the present retrospective study may fail to provide an estimation of the prevalence of canine angiostrongylosis in Belgium since only dogs with respiratory clinical signs (i.e. cough and/or dyspnoea) were considered and patients with other clinical signs, such as cardiac and central nervous system involvement or coagulopathy, were not considered. Besides, inclusion of large populations of healthy dogs would have been also required because asymptomatic infection can be detected in only $<2 \%$ of pets dogs in some endemic areas (Elsheikha et al. 2014).

Although bronchoscopy and bronchoalveolar lavage are fairly safe procedures, the main disadvantage for collecting bronchoalveolar lavage fluid in coughing dogs is the need for anaesthesia, which can be particular risky in some dyspnoeic dogs with angiostrongylosis. Inherently safer tests are currently available to help to make a diagnosis (Baermann examination or in-house rapid device for circulating antigen) and should be included in the diagnostic approach as first-line tests, even though they have drawbacks (especially sensitivity) as previously alluded to. The financial cost of bronchoscopy may also limit its use as a first choice diagnostic procedure. Therefore, it would be interesting to prospectively compare the respective diagnostic values of qPCR performed on various samples (broncho-alveolar lavage fluid, faeces, blood) and the non-invasive in-house device for antigen testing, in a series of coughing or dyspnoeic dogs. Based on the present findings, qPCR on broncho-alveolar lavage fluid is a valuable diagnostic tool and should, at least, be recommended for dogs clinically suspected to have pulmonary angisotrongylosis and that have negative result for Baermann analysis and/or negative result for rapid antigen testing on blood.

Furthermore, until this present study, endoscopy findings in dogs with angiostrongylosis were uncommonly described in detail. Based on our observations, any detection of fresh blood or blood-containing secretions in lower airways during bronchoscopy of coughing or dyspneic dogs should lead clinicians to have the suspicion of angiostrongylosis.

In conclusion, based on results of Baermann analysis on faeces and qPCR on broncho-alveolar lavage fluid, pulmonary angiostrongylosis in coughing dogs was negligible in Belgium until 2013 but should now be considered as an emerging and endemic condition and included in the differential diagnosis of coughing Belgian dogs of any age, especially when blood-containing bronchial secretions are detected on bronchoscopy.

\section{Conflict of interest}

None of the authors of this article have a financial or personal relationship with other people or organisations that could inappropriately influence or bias the content of this paper.

\section{References}

Barçante, J. M., Barçante, T. A., Ribeiro, V. M., et al. (2008) Cytological and parasitological analysis of bronchoalveolar lavage fluid for the diagnosis of Angiostrongylus vasorum infection in dogs. Veterinary Parasitology 25, 93-102

Barutzki, D. \& Schaper, R. (2009) Natural infections of Angiostrongylus vasorum and Crenosoma vulpis in dogs in Germany (2007-2009). Parasitology Research 105 (Suppl 1), S39-S48

Bolt, G., Monrad, J., Henriksen, P., et al. (1992) The fox as a reservoir for canine angiostrongylosis in Denmark. Field survey and experimental infections. Act Veterinaria Scandinavica 33, 352-362

Bolt, G., Monrad, J., Koch, J., et al. (1994) Canine angiostrongylosis: a review. Veterinary Record 135, 447-452
Bourque, A. C., Conboy, G., Miller, L. M., et al. (2008) Pathological findings in dogs naturally infected with Angiostrongylus vasorum in Newfoundland and Labrador, Canada. Journal of Veterinary Diagnostic Investigation 20, 11-20

Chapman, P. S., Boag, A. K., Guitian, J., et al. (2004) Angiostrongylus vasorum infection in 23 dogs (1999-2002). Journal of Small Animal Practice 45, 435-440

Conboy, G. A. (2011) Canine angiostrongylosis: the French heartworm: an emerging threat in North America. Veterinary Parasitology 176, 382-389

Dehard, S., Bernaerts, F., Peeters, D., et al. (2008) Comparison of bronchoalveolar lavage cytospins and smears in dogs and cats. Journal of the American Animal Hospital Association 44, 285-294

Demiaszkiewicz, A. W., Pyziel, A. M., Kuligowska, I., et al. (2014) The first report of Angiostrongylus vasorum in Poland, in red foxes (Vulpes vulpes). Acta Parasitologica 59, 758-762

Doby, J. M., Drozdz, J. \& Beaucourmu, J. C. (1970) Existence in France of Angiostrongylus (Metastrongyloidea, Nematoda, a parasite of wild rodents). Bulletin de la Societe de Pathologie Exotique et de Ses Filiales 63, 376-381

Elsheikha, H. M., Holmes, S. A, Wright, I., et al. (2014) Recent advances in the epidemiology, clinical and diagnostic features, and control of canine cardiopulmonary angiostrongylosis. Veterinary Research 45, 92

Forey, W. J. (1989) Diagnostic parasitology. Veterinary Clinics of North America: Small Animal Practice 19, 979-1000

Franssen, F., Nijsse, R., Mulder, J., et al. (2014) Increase in number of helminth species from Dutch red foxes over a 35-year period. Parasites \& Vectors 3, 166

Gallagher, B., Brennan, S. F., Zarelli M., et al. (2012) Geographical, clinical, clinicopathological and radiographic features of canine angiostrongylosis in Irish dogs: a retrospective study. Irish Veterinary Journal 20, 5

Gredal, H., Willesen, J., Jensen, H., et al. (2011) Acute neurological signs as the predominant clinical manifestation in four dogs with Angiostrongylus vasorum infections in Denmark. Acta Veterinaria Scandinavica 53, 43-50

Guardone, L., Schnyder, M., Macchioni, F., et al. (2013) Serological detection of circulating Angiostrongylus vasorum antigen and specific antibodies in dogs from central and northern Italy. Veterinary Parasitology 18, 192-198

Helm, J. R., Morgan, E.R., Jackson, M. W., et al. (2010) Canine angiostrongylosis: an emerging disease in Europe. Journal of Veterinary Emergency and Critical Care 20, 98-109

Humm K. \& Adamantos S. (2010) Is evaluation of a faecal smear a useful technique in the diagnosis of canine pulmonary angiostrongylosis ? Journal of Small Animal Practice 51, 200-203

Jefferies, R., Morgan, E. R., Helm, J., et al. (2011) Improved detection of canine Angiostrongylus vasorum infection using real-time PCR and indirect ELISA. Parasitology Research 109, 1577-1583

Jeffery, R. A., Lankester, M. W., Mcgrath, M. J., et al. (2004) Angiostrongylus vasorum and Crenosoma vulpis in red foxes (Vulpes vulpes) in Newfoundland, Canada. Canadian Journal of Zoology 82, 66-74

Jolly, S., Poncelet, L., Lempereur, L., et al. (2014) First report of a fatal autochthonous canine Angiostrongylus vasorum infection in Belgium. Parasitology International 29, 97-99

Koch, J. \& Willesen, J. L. (2009) Canine pulmonary angiostrongylosis: an update. Veterinary Journal 179, 348-359

Morgan, E. R., Jefferies, R., Krajewski, M., et al. (2009) Canine pulmonary angiostrongylosis: the influence of climate on parasite distribution. Parasitology International 58, 406-410

Oliveira-Junior, S. D., Barçante, J. M., Barçante, T. A., et al. (2006) Larval output of infected and re-infected dogs with Angiostrongylus vasorum (Baillet, 1866) Kamensky, 1905. Veterinary Parasitology 141, 101-106

Schnyder, M., Tanner, I., Webster, P., et al. (2011) An ELISA for sensitive and specific detection of circulating antigen of Angiostrongylus vasorum in serum samples of naturally and experimentally infected dogs. Veterinary Parasitology 30, 152-158

Schnyder, M., Schaper, R., Bilbrough, G., et al. (2013) Seroepidemiological survey for canine angiostrongylosis in dogs from Germany and the UK using combined detection of Angiostrongylus vasorum antigen and specific antibodies. Parasitology 140, 1442-1450

Schnyder, M., Stebler, K., Naucke, T. J., et al. (2014) Evaluation of a rapid device for serological in-clinic diagnosis of canine angiostrongylosis. Parasites \& Vectors 18, 72

Schucan, A., Schnyder, M. Tanner, I., et al. (2012) Detection of specific antibodies in dogs infected with Angiostrongylus vasorum. Veterinary Parasitology 30, 216-224

Taubert, A., Pantchev, N., Vrhovec, M. G., et al. (2009) Lungworm infections (Angiostrongylus vasorum, Crenosoma vulpis, Aelurostrongylus abstrusus) in dogs and cats in Germany and Denmark in 2003-2007. Veterinary Parasitology 159, 175-180

Traversa, D., Di Cesare, A., Conboy, G. (2010) Canine and feline cardiopulmonary parasitic nematodes in Europe: emerging and underestimated. Parasit Vectors 3, 62

Traversa, D., Di Cesare, A., Meloni, S., et al. (2013) Canine angiostrongylosis in Italy: occurrence of Angiostrongylus vasorum in dogs with compatible clinical pictures. Parasitology Research 112, 2473-2480

Van Doorn, D., Van de Sande, A., Nijsse, E., et al. (2009) Autochthonous Angiostrongylus vasorum infection in dogs in The Netherlands. Veterinary Parasitology 162, 163-166

Verzberger-Epshtein, I., Markham, R. J., Sheppard, J. A., et al. (2008) Serologic detection of Angiostrongylus vasorum infection in dogs. Veterinary Parasitology 151, 53-60

Yamakawa, Y., Mcgarry, J. W., Denk, D., etal. (2009) Emerging canine angiostrongylosis in northern England: five fatal cases. Veterinary Record 164, 149-152 